\title{
RAPID SPREAD OF DRUG-RESISTANT INFLUENZA A VIRUSES in the Basque Country, northern Spain, 2000-1 to $2008-9$
}

\author{
D Vicente ${ }^{1,2}$, G Cilla1,2, M Montes ${ }^{1,2}$, J Mendiola1, E Pérez-Trallero (mikrobiol@terra.es) \\ 1. Microbiology Service and Reference Laboratory for Influenza Infections of the Basque Country, Hospital Donostia, San \\ Sebastián, Spain \\ 2. Biomedical Research Centre Network for Respiratory Diseases (CIBERES), San Sebastián, Spain \\ 3. Department of Preventive Medicine and Public Health, Faculty of Medicine, University of the Basque Country, San Sebastián, \\ Spain
}

\begin{abstract}
A worldwide increase of adamantane-resistant influenza A(H3N2) and oseltamivir-resistant influenza $A(\mathrm{H} 1 \mathrm{~N} 1)$ viruses has been observed in recent years. The aim of this study was to analyse the prevalence of antiviral drug-resistant influenza $A$ in a region of northern Spain. Resistance to adamantanes was detected in $45.3 \%$ (68/150) of influenza AH3 viruses analysed for the period from 2000-1 to 2008-9. Adamantane-resistance was absent in our region during the 2000-1 to 2002-3 influenza seasons. However, after the first adamantane-resistant virus (characterised as A/ Fujian/411/2002) was detected in the 2003-4 season, a rapid increase in the proportion of resistant strains was observed $(4.9 \%$ [2/41], 80\% [8/10] and 100\% [53/53] in the 2004-5, 2006-7 and 2008-9 seasons, respectively). Four of the first five adamantaneresistant $\mathrm{AH} 3$ viruses detected were isolated from adult patients, but the subsequent spread was observed mainly among children. No resistance to adamantanes was detected among the 65 influenza $\mathrm{AH} 1$ viruses analysed throughout the study period. Among the 172 influenza $\mathrm{A}$ (76 $\mathrm{AH} 1$ and $96 \mathrm{AH} 3$ ) viruses analysed, five strains ( $\mathrm{AH} 1$ with mutation $\mathrm{H} 274 \mathrm{Y}$ ) showed oseltamivir resistance, and all were detected in the last season. Amantadine use was very scarce in our region, and oseltamivir was not used at all; therefore the increase of resistance was attributed to imported drug-resistant influenza viruses.
\end{abstract}

\section{Introduction}

Only four licensed influenza antiviral agents are currently available: the adamantanes - amantadine and rimantadine, and the neuraminidase inhibitors - zanamivir and oseltamivir. Amantadine hydrochloride was approved in the United States (US) in 1966 for chemoprophylaxis of influenza $\mathrm{A}(\mathrm{H} 2 \mathrm{~N} 2)$ and has been used to prevent and treat influenza $A$ for more than three decades [1]. Although adamantanes, which block the function of the M2 protein, can reduce the severity and duration of influenza $A$ infection in healthy adults, their use has been limited due to rapid selection of resistant viruses during treatment. In recent years, a high percentage of influenza $A(H 3 N 2)$ viruses circulating in Asia, America and eastern Europe have shown resistance to adamantanes [2]. Southern Europe seems to have escaped this problem but resistance to oseltamivir has been observed since the beginning of the 2007-8 influenza season among influenza $A(H 1 N 1)$ viruses [3].
The aim of this study was to determine the prevalence of resistance to adamantanes and neuraminidase inhibitors (oseltamivir and zanamivir) in influenza $A$ isolates obtained during nine seasons (2000-1 to 2008-9) in the Basque Country, northern Spain, a region that borders the southwest of France.

\section{Materials and methods}

The study was performed in the Microbiology Department of Hospital Donostia, which is the Reference Laboratory for Influenza Infections in the Basque Country, and has been integrated in the Spanish Influenza Surveillance System since 1998. Of the available 587 respiratory samples that tested positive by cell culture for influenza A virus, 282 (48\%) were selected for the susceptibility study. All the minority subtype strains and an unselected sample of the predominant subtype circulating in each season were included. Most of the strains included in the study were obtained from consecutive patients who had consulted physicians participating in the Spanish Sentinel Influenza Surveillance System; a smaller proportion (19\%) of isolates studied were obtained from patients admitted to or treated in the emergency department of our hospital. The age and gender of patients included in this study represent the normal distribution of people with influenza in our region. An aliquot of $400 \mu \mathrm{L}$ of all the original samples was conserved at $-80^{\circ} \mathrm{C}$ until susceptibility studies were performed. The distinct number of clinical samples studied in each season was dependent on differences in the season-to-season dynamics of influenza $A$ viruses. The exact numbers of isolates of $\mathrm{AH} 1$ and $\mathrm{AH} 3$ viruses tested in each season and for each drug class are listed in the Table. The resistant strains mentioned in this study have not previously been reported in any other publication, except for four $\mathrm{AH} 1$ oseltamivirresistant strains reported to the European Influenza Surveillance Scheme (http://www.eiss.org/).

Genotypic resistance was detected by sequencing of viral genome fragments and identification of mutations previously associated with drug-resistance. Viral RNA was extracted from respiratory samples using the bioMérieux NucliSENS easyMAG system (bioMérieux, Marcy l'Etoile, France). Transcription of RNA to cDNA was performed with M-MuLVreverse transcriptase (Promega, Madison, WI, US) using random primers. An M2 gene fragment (330 bp) $[4,5]$ and a neuraminidase gene fragment 
(708 bp) [6,7] were amplified for analysis of adamantane and neuraminidase inhibitor susceptibility, respectively. Amplified gene fragments were sequenced in an ABI PRISM 3100 Genetic Analyser (Applied Biosystems, Foster City, CA, US) and amino acid-deduced sequences were obtained. M2 and neuraminidase sequences were analysed to identify mutations previously associated with antiviral resistance. The amino acid substitutions L26F, V27A, A30T, S31N and G34E in the M2 protein were associated with resistance to adamantanes [4,8], the amino acid substitutions H274Y, E119V and $\mathrm{R} 292 \mathrm{~K}$ in the neuraminidase protein were associated with resistance to oseltamivir, and substitutions $\mathrm{Y} 155 \mathrm{H}$ and $1222 \mathrm{~T}$ with resistance to zanamivir [9]. Phylogenetic analysis of the haemagglutinin gene was performed at the Reference Center for Influenza Surveillance in Spain (Instituto de Salud Carlos III).

\section{Results}

Mutations conferring resistance to adamantanes were detected in $31.6 \%(68 / 215)$ of the influenza A viruses studied over nine seasons (2000-1 to 2008-9). Resistance to adamantanes was detected in $45.3 \%(68 / 150)$ of influenza AH3 viruses, while no influenza $\mathrm{AH} 1$ viruses with mutations conferring resistance were found $(0 / 65)$ (Table).

The first case of adamantane-resistance was detected in a sample obtained in November 2003 from a 37-year-old woman with typical influenza symptoms (high temperature, headache, muscle ache and respiratory symptoms). Phylogenetic analysis of the haemagglutinin gene confirmed its similarity with the $A /$ Fujian/411/2002 strain. The proportion of strains resistant to adamantanes among AH3 viruses was 0\% (0/20) from 2000-1 to 2002-3 season, $7.9 \%$ (5/63) from 2003-4 to 2005-6 season and $94 \%(63 / 67)$ during the last three seasons (Table, Figure). Four of the first five cases of adamantane-resistant $\mathrm{AH} 3$ viruses were detected in young adults ( 25 to 47 years old), but the subsequent eight cases were detected in children. Throughout the study period, resistance to adamantanes was more frequently detected among children $(32 / 80,40 \%)$ than adults $(36 / 135,26.7 \%)(P<0.05)$. All adamantane-resistant strains except one showed serine-toasparagine (S31A) amino acid substitution at position 31 . The remaining strain showed glycine-to-glutamic acid (G34E) amino acid substitution at position 34 .

Among 172 influenza $\mathrm{A}$ (76 $\mathrm{AH} 1$ and $96 \mathrm{AH} 3$ ) isolates analysed for resistance to oseltamivir, five strains $(\mathrm{AH} 1$ with mutation $\mathrm{H} 274 \mathrm{Y}$ ) were found resistant. All of these were characterised as A/Brisbane/59/2007 (H1N1)-like AH1. The first was isolated in November 2008 from an 18-year-old man with typical influenza symptoms. Resistance to zanamivir was not detected in any of the 172 isolates studied.

\section{Discussion}

The results of this study show that, unlike the situation before 2004-5, most of the influenza AH3 virus strains currently circulating in our region are resistant to adamantanes $(100 \%$

\section{F I G U R E}

Triannual distribution of adamantane-resistant influenza AH3 virus strains detected between the 2000-1 and 2008-9 seasons in the Basque Country, northern Spain

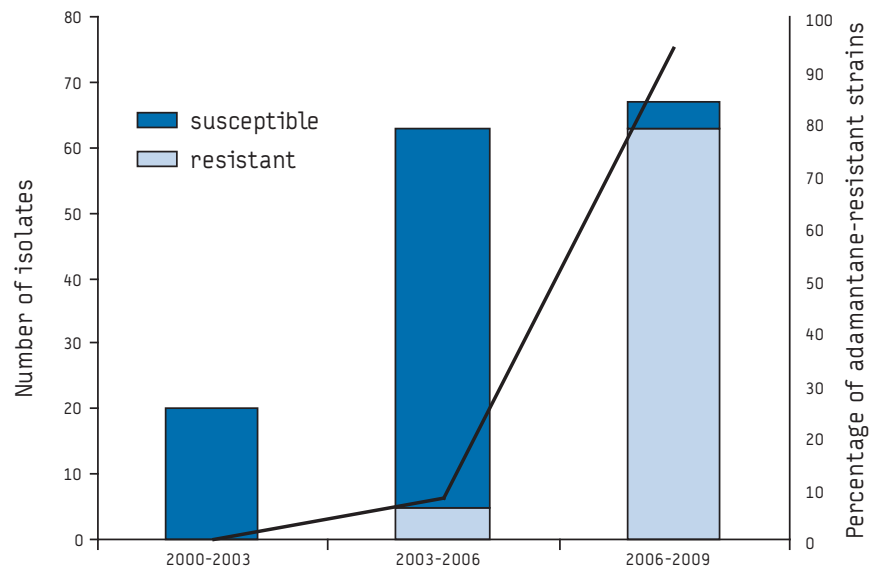

Number of influenza A viruses included in the genetic analysis of antiviral resistance during nine influenza seasons (2000-1 to 2008-9) in the Basque Country, northern Spain.

\begin{tabular}{|c|c|c|c|c|c|}
\hline \multirow{3}{*}{ Season } & \multicolumn{2}{|c|}{ Adamantanes } & \multicolumn{2}{|c|}{ Oseltamivir* } & \multirow{3}{*}{ Predominant virus in the season } \\
\hline & AH1 & AH3 & AH1 & AH3 & \\
\hline & investigated/resistant & investigated/resistant & investigated/resistant & investigated/resistant & \\
\hline $2000-1$ & $7 / 0$ & $1 / 0$ & $1 / 0$ & $0 / 0$ & $A / B$ \\
\hline 2001-2 & $3 / 0$ & $18 / 0$ & $0 / 0$ & $7 / 0$ & $\mathrm{~A}(\mathrm{H} 3 \mathrm{~N} 2) / \mathrm{B}$ \\
\hline $2002-3$ & $13 / 0$ & $1 / 0$ & $3 / 0$ & $0 / 0$ & $\mathrm{~B} / \mathrm{A}(\mathrm{H} 1 \mathrm{~N} 1)$ \\
\hline 2003-4 & $0 / 0$ & $18 / 1$ & $0 / 0$ & $2 / 0$ & $\mathrm{~A}(\mathrm{H} 3 \mathrm{~N} 2)$ \\
\hline $2004-5$ & $0 / 0$ & $41 / 2$ & $0 / 0$ & $5 / 0$ & $\mathrm{~A}(\mathrm{H} 3 \mathrm{~N} 2)$ \\
\hline $2005-6$ & $19 / 0$ & $4 / 2$ & $8 / 0$ & $0 / 0$ & $\mathrm{~A}(\mathrm{H} 1 \mathrm{~N} 1) / \mathrm{B}$ \\
\hline $2006-7$ & $0 / 0$ & $10 / 8$ & $0 / 0$ & $20 / 0$ & $\mathrm{~A}(\mathrm{H} 3 \mathrm{~N} 2)$ \\
\hline $2007-8$ & $18 / 0$ & $4 / 2$ & $59 / 0$ & $9 / 0$ & $\mathrm{~A}(\mathrm{H} 1 \mathrm{~N} 1) / \mathrm{B}$ \\
\hline $2008-9$ & $5 / 0$ & $53 / 53$ & $5 / 5$ & $53 / 0$ & $\mathrm{~A}(\mathrm{H} 3 \mathrm{~N} 2)$ \\
\hline Total & $65 / 0$ & $150 / 68$ & $76 / 5$ & $96 / 0$ & \\
\hline
\end{tabular}

* Resistance to zanamivir was not detected in any of the 172 influenza A viruses studied 
resistance in the 2008-9 season). In 2005, genetic studies confirmed the emergence of adamantane-resistant influenza AH3 strains in China and Hong Kong [10]. In the United States, the frequency of adamantane-resistance increased from 1.9\% during the $2003-4$ influenza season to $11 \%$ during the $2004-5$ season [11]. Since then, a growing number of resistant $\mathrm{AH} 3$ viruses have been reported in several countries, with $100 \%$ resistance reached in some Asian countries [2]. In most cases, the amino acid substitution detected (S31N) was the same as that detected in the present study. Anti-M2-resistant strains easily emerge during treatment with adamantanes [12]. Rimantadine is not licensed in Spain, while amantadine is available on prescription only, is not included in any over-the-counter cold remedies, and its use in our region is scarce. The number of defined daily doses per 1,000 inhabitants per day [13] of amantadine in 2007 in this region, which has approximately 2 million inhabitants, was 0.13 and was mainly limited to the treatment of Parkinson's disease. Therefore, the high resistance rate detected in our region is probably due to importation of resistant strains from other areas. Although the first few cases occurred in adults, the full spread across the region occurred mainly through children.

In January 2008, the emergence of resistance to oseltamivir among influenza $A(\mathrm{H} 1 \mathrm{~N} 1)$ viruses was reported in Europe [3]. The results of analysis of early winter isolates revealed that $20 \%$ of the European strains were resistant to oseltamivir but retained sensitivity to zanamivir and adamantanes [3,14]. Up to June 2008, 52 countries worldwide reported similar results. The viruses carried a specific neuraminidase mutation ( $\mathrm{H} 274 \mathrm{Y}$ ) that confers highlevel resistance to oseltamivir in N1-containing influenza viruses $[3,9,15]$. Despite the spread of resistance across Europe, in Spain only two out of $108(1.9 \%) \mathrm{A}(\mathrm{H} 1 \mathrm{~N} 1)$ strains previously studied showed the H274Y mutation [14]. In our study, no mutations conferring neuraminidase inhibitor resistance were detected among the influenza A viruses (43 $\mathrm{AH} 3$ and $71 \mathrm{AH} 1$ strains) analysed between the 2000-1 and 2007-8 seasons. However, during the 2008-9 season of the five influenza AH1 strains isolated, all five showed the $\mathrm{H} 274 \mathrm{Y}$ mutation conferring oseltamivir resistance. Neither the patients nor their closest contacts had received oseltamivir treatment, which suggested that, as occurred with the first adamantane-resistant viruses, these viruses were already resistant before infecting these patients. The north of our region flanks the border with France, where $46.6 \%$ of the $A(H 1 N 1)$ viruses studied during the 2007-8 season showed oseltamivir resistance [14].

The present study reveals, once again, how resistance can appear in a region without prior pressure from antiviral drugs and how resistant strains can rapidly disseminate among the population. In addition to promoting influenza vaccination among the general population, research into new anti-influenza agents that could counteract the effects of this resistance should be stimulated.
3. Lackenby A, Hungnes O, Dudman SG, Meijer A, Paget WJ, Hay AJ, et al. Emergence of resistance to oseltamivir among influenza A(H1N1) viruses in Europe. Euro Surveill. 2008;13(5):pii=8026. Available from: http://www.eurosurveillance.org/ ViewArticle. aspx? ArticleId $=8026$

4. Saito $R$, Oshitani $H$, Masuda $H$, Suzuki $H$. Detection of amantadine-resistant influenza A virus strains in nursing homes by PCR-restriction fragment length polymorphism analysis with nasopharyngeal swabs. J Clin Microbiol. 2002;40(1):84-8.

5. Chan CH, Lin KL, Chan Y, Wang YL, Chi YT, Tu HL, et al. Amplification of the entire genome of influenza A virus H1N1 and H3N2 subtypes by reverse-transcription polymerase chain reaction. J Virol Methods. 2006;136(1-2):38-43.

6. Takao S, Shimazu Y, Fukuda S, Kuwayama M, Miyazaki K. Neuraminidase subtyping of human influenza a viruses by RT-PCR and its application to clinical isolates. Jpn J Infect Dis. 2002;55(6):204-5.

7. Mak A, Rahmanian R, Lei V, Lawrence D, Krajden M, Brunham RC, et al. Longitudinal analysis of genotype distribution of influenza A virus from 2003 to 2005.J Clin Microbiol. 2006;44(10):3583-8.

8. Saito R, Sakai T, Sato I, Sano Y, Oshitani H, Sato M, et al. Frequency of amantadine-resistant influenza A viruses during two seasons featuring cocirculation of H1N1 and H3N2. J Clin Microbiol. 2003;41(5):2164-5.

9. Monto AS, McKimm-Breschkin JL, Macken C, Hampson AW, Hay A, Klimov A, et al. Detection of influenza viruses resistant to neuraminidase inhibitors in global surveillance during the first 3 years of their use. Antimicrob Agents Chemother. 2006;50(7):2395-402.

10. Bright RA, Medina MJ, Xu X, Perez-Oronoz G, Wallis TR, Davis XM, et al. Incidence of adamantane resistance among influenza $A(H 3 N 2)$ viruses isolated worldwide from 1994 to 2005: a cause for concern. Lancet. 2005;366(9492):117581.

11. Centers for Disease Control and Prevention (CDC). High levels of adamantane resistance among influenza A (H3N2) viruses and interim guidelines for use of antiviral agents--United States, 2005-06 influenza season. MMWR Morb Mortal Wkly Rep. 2006;55(2):44-6.

12. Englund JA, Champlin RE, Wyde PR, Kantarjian H, Atmar RL, Tarrand J, et al. Common emergence of amantadine- and rimantadine-resistant influenza A viruses in symptomatic immunocompromised adults. Clin Infect Dis. 1998;26(6):1418-24.

13. WHO Collaborating Centre for Drug Statistics Methodology Anatomic Therapeutic Chemical (ATC) classification index with Defined Daily Doses (DDDs). WHO Collaborating Centre for Drug Statistics Methodology: Oslo, Norway; 2003. Available from: http://www.whocc.no/atcddd

14. Meijer A, Lackenby A, Hungnes O, Lina B, van-der-Werf S, Schweiger B, et al. Oseltamivir-resistant influenza virus A (H1N1), Europe, 2007-8 season. Emerg Infect Dis. 2009;15(4):552-560.

15. de Jong MD, Tran TT, Truong HK, Vo MH, Smith GJ, Nguyen VC, et al. Oseltamivir resistance during treatment of influenza A (H5N1) infection. N Engl J Med. 2005;353(25):2667-72.

This article was published on 21 May 2009.

Citation style for this article: Vicente D, Cilla G, Montes M, Mendiola J, Pérez-Trallero E. Rapid spread of drug-resistant influenza A viruses in the Basque Country, northern Spain, 2000-1 to 2008-9. Euro Surveill. 2009;14(20):pii=19215. Available online: http:// www.eurosurveillance.org/ViewArticle.aspx?ArticleId=19215

\section{References}

1. Harper SA, Fukuda K, Uyeki TM, Cox NJ, Bridges CB; Advisory Committee on Immunization Practices (ACIP), Centers for Disease Control and Prevention (CDC). Prevention and control of influenza. Recommendations of the Advisory Committee on Immunization Practices (ACIP). MMWR Recomm Rep. 2005;54(RR8):1-40.

2. Deyde VM, Xu X, Bright RA, Shaw M, Smith CB, Zhang Y, et al. Surveillance of resistance to adamantanes among influenza $A(H 3 N 2)$ and $A(H 1 N 1)$ viruses isolated worldwide. J Infect Dis. 2007;196(2):249-57. 